\title{
Trans-sigmoidal endoscopic ultrasonography-guided fine needle biopsy of a pan- creas allograft for the diagnosis of post-transplant lymphoproliferative disorder
}

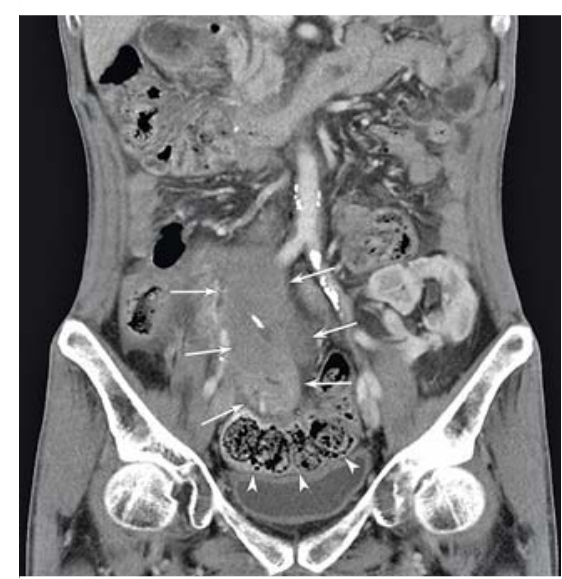

- Fig. 1 Abdominopelvic computed tomography (CT) scan showing diffuse swelling and heterogeneous attenuation of the pancreas allograft (white arrows). and its close anatomical relation to the sigmoid colon (white arrowheads).

Biopsy of pancreas allografts has a critical role in the evaluation of pancreas allograft dysfunction [1]. However, percutaneous ultrasound or computed tomography (CT)-guided biopsy of a pancreas allograft in the pelvis may not be feasible in some cases. Pelvic lesions are easily imaged endosonographically from the rectum or distal sigmoid colon. Although transrectal endoscopic ultrasonography (EUS)-guided biopsy or drainage for pelvic lesions has been demonstrated to be feasible and safe [2,3], there have been no reports of lower gastrointestinal EUSguided fine needle biopsy (FNB) of a pancreas allograft in the pelvis owing to the unique anatomic configuration of the post-transplant anatomy [4].

A 47-year-old man underwent simultaneous pancreas and kidney transplantation 10 years ago for insulin-dependent diabetes mellitus and end-stage renal disease due to diabetic nephropathy. The graft duodenum had been anastomosed to the proximal jejunum to allow for enteric exocrine drainage. The patient presented to us with right lower abdominal pain and elevated levels of amy-

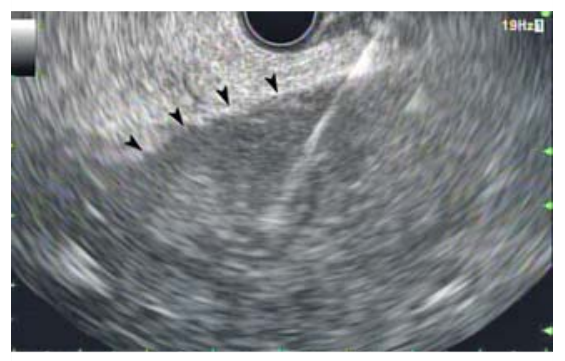

- Fig. 2 Image during endoscopic ultrasonography-guided fine needle biopsy (EUS-FNB) of the pancreas allograft (black arrowheads).

lase and lipase. His abdominopelvic CT scan showed swelling of the pancreas allograft with pancreatic duct dilatation and peripancreatic infiltration ( $>$ Fig. 1). Given this picture, histologic evaluation of the pancreas allograft was mandatory, but the direction of its body and tail, placed towards the right pelvis, was not suitable for percutaneous access. Therefore, we decided to perform a trans-sigmoidal EUS-guided FNB.

The procedure was performed after the patient had undergone bowel preparation. A linear echoendoscope was rotated counter-clockwise in the distal sigmoid colon, and the pancreas allograft was visualized in the right pelvis. Trans-sigmoidal EUS-FNB was performed with a 22-gauge needle (ProCore Needle; COOK Medical) (> Fig. 2; > Video 1) using the stylet slow-pull technique for a pancreas allograft. A total of four needle passes were made and no procedure-related adverse events were seen.

The biopsy revealed monomorphic-type post-transplant lymphoproliferative disorder consistent with Burkitt lymphoma based on histological, immunohistochemical, and fluorescence in situ hybridization (FISH) analysis ( $\triangleright$ Fig.3). After receiving the first cycle of hyper-fractionated cyclophosphamide, vincristine, doxorubicin, and dexamethasone (Hyper-CVAD), the patient's lower abdominal pain and elevated pancreatic enzymes were improved.

Endoscopy_UCTN_Code_CCL_1AF_2AG_3AD

Competing interests

None

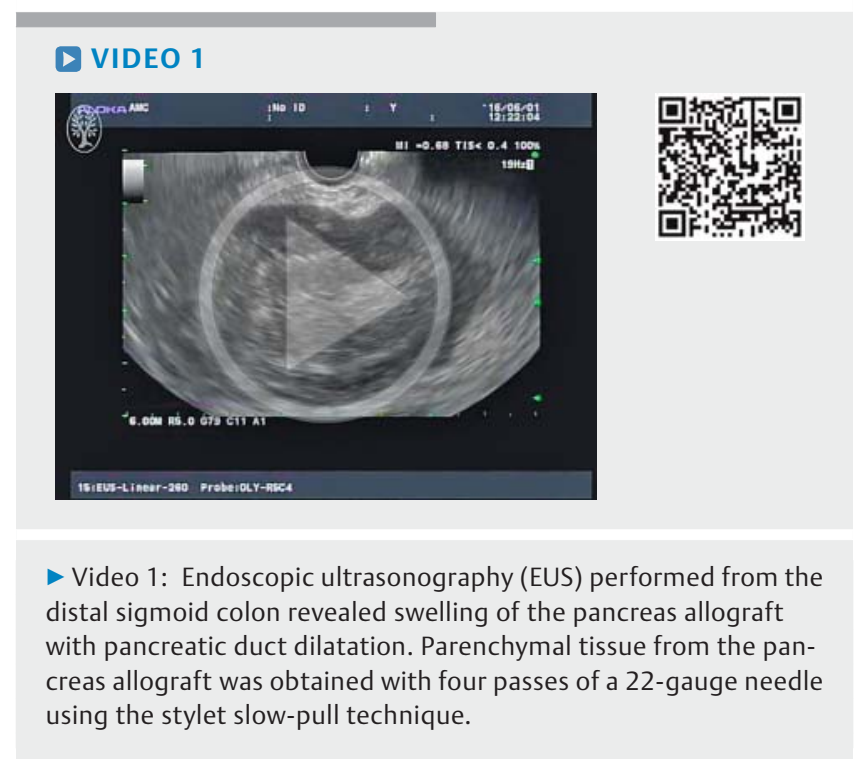




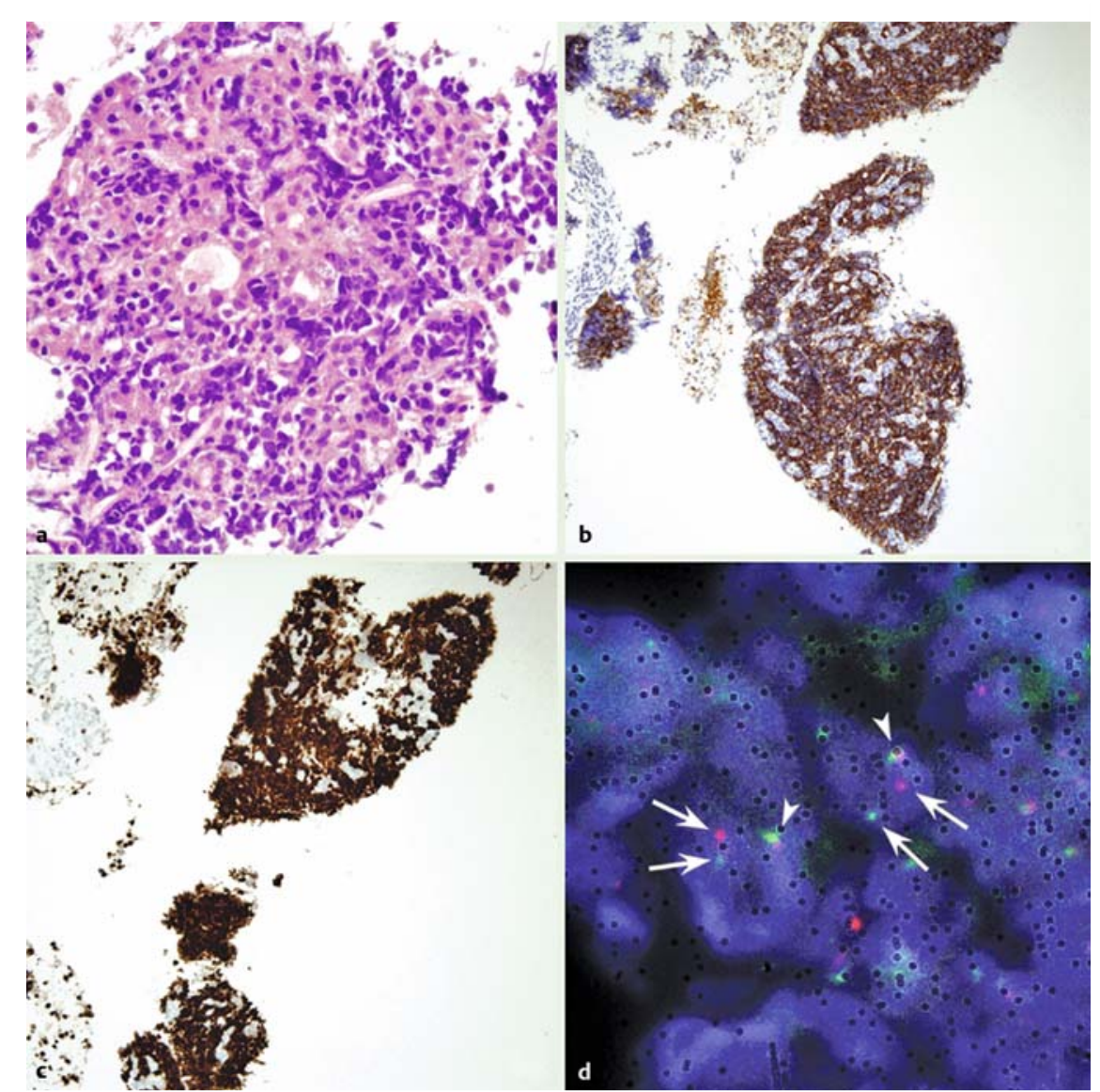

- Fig.3 Representative images of the pancreas allograft biopsy. a Histological appearance showing a diffuse infiltration of monomorphic medium-sized tumor cells on hematoxylin and eosin (H\&E) staining (magnification $\times 400)$. b, c Immunohistochemical staining (magnification $\times 100$ ) with: $\mathbf{b}$ CD20 showing that the tumor cells were of B-cell origin; $\mathbf{c}$ Ki- 67 showing $>99 \%$ of tumor cells were in proliferation. $\mathbf{d}$ Fluorescence in situ hybridization (FISH) analysis showing the c-MYC rearrangement detected by a dual-color, break-apart probe as indicated by the split green and red signals (white arrows) in comparison with unaffected signals (white arrowheads).

[1] Gaber LW. Pancreas allograft biopsies in the management of pancreas transplant recipients: Histopathologic review and clinical correlations. Arch Pathol Lab Med 2007; 131: $1192-1199$

[2] Hassan GM, Paquin SC, Albadine R et al. Endoscopic ultrasound-guided FNA of pelvic lesions: A large single-center experience. Cancer Cytopathol 2016. DOI: 10.1002/ cncy. 21756

[3] Varadarajulu S, Lee YT, Group EUSW. EUS 2008 Working Group document: evaluation of EUS-guided drainage of pelvic-fluid collections (with video). Gastrointest Endosc 2009; 69: 32-\$36

[4] Horneland R, Paulsen V, Lindahl JP et al. Pancreas transplantation with enteroanastomosis to native duodenum poses technical challenges but offers improved endoscopic access for scheduled biopsies and therapeutic interventions. Am J Transplant 2015; $15: 242-250$

\section{Bibliography}

DOI http://dx.doi.org/10.1055/s-0042-119397

Endoscopy 2017; 49: E14-E15

(c) Georg Thieme Verlag KG

Stuttgart - New York

ISSN 0013-726X

\section{The Authors}

Hyungil Seo ${ }^{1,{ }^{*}}$, Dong Hui Cho ${ }^{1,{ }^{*}}$, Sun-Ho Lee ${ }^{1}$, Jin Ho Shin ${ }^{2}$, Young Hoon Kim³ ${ }^{3}$ Do Hyun Park ${ }^{1}$

1 Department of Gastroenterology, University of Ulsan College of Medicine, Asan Medical Center, Seoul, Republic of Korea

2 Department of Surgery, University of Ulsan College of Medicine, Asan Medical Center, Seoul, Republic of Korea

3 Department of Pathology, University of Ulsan College of Medicine, Asan Medical Center, Seoul, Republic of Korea

\section{Corresponding author}

\section{Do Hyun Park, MD, PhD}

Department of Gastroenterology, University of Ulsan College of Medicine, Asan Medical Center, 88, Olympic-ro 43-gil, Songpa-gu, Seoul, 05505, Republic of Korea,

Fax: +82-2-4760824,

dhpark@amc.seoul.kr

* contributed equally to this work 